\title{
Wave topology brought to the coast
}

\author{
A. Venaille (1) ${ }^{*}$ and P. Delplace ${ }^{\dagger}$ \\ Univ Lyon, ENS de Lyon, Univ Claude Bernard, CNRS, Laboratoire de Physique, F-69342 Lyon, France
}

(Received 29 March 2021; accepted 23 August 2021; published 1 October 2021)

\begin{abstract}
Since the pioneering work of Kelvin on Laplace tidal equations, a zoology of trapped waves have been found in the context of coastal dynamics. Among them, the one originally computed by Kelvin plays a particular role, as it is a unidirectional mode filling a frequency gap between different wave bands. The existence of such Kelvin waves is robust to changes in the boundary shape and in changes of the underlying model for the coast. This suggests a topological interpretation that has yet up to now remained elusive. Here we rectify the situation, by taking advantage of a reformulation of the shallow water dynamics that highlights an analogy with the celebrated Haldane model in condensed matter physics. For any profile of bottom topography, the number of modes that transit from one wave band to another in the dispersion relation is predicted by computing a first Chern number describing the topology of complex eigenmodes in a dual, simpler wave problem.
\end{abstract}

DOI: 10.1103/PhysRevResearch.3.043002

\section{INTRODUCTION}

Coastal Kelvin waves were derived in 1880 in an attempt to solve tidal Laplace equations in oceanic basins [1]. These equations model the linear dynamics of surface shallow water in the presence of the Coriolis force. Kelvin waves are trapped modes that have the noteworthy properties to fill a frequency gap between different wavebands and to travel as surface waves in a cyclonic manner along the coasts. Such waves are now routinely observed along continental margins or sufficiently large lakes.

These remarkable features bear striking similarities with peculiar electronic states found over the last decades at the boundary of exotic materials called topological insulators, and more specifically Chern insulators. In such material, the number of unidirectional trapped waves along the boundary is ruled by a single number, the first Chern number, that describes the topology of normalized eigenmode bundles in an abstract dual bulk problem. This bulk problem describes similar waves, but in unbounded geometries and with homogeneous coefficients in the linear operator. The first Chern number of the abstract bulk problem predicts the number of unidirectional boundary trapped modes in the original wave problem through a celebrated bulk-boundary correspondence [2]. Such boundary trapped modes are often said to have a topological origin, or to be topologically protected.

Topology offers a powerful tool to make predictions on a complicated problem without having to solve this problem. Here, robust properties of partial differential equations with

\footnotetext{
*antoine.venaille@ens-lyon.fr

†pierre.delplace@ens-lyon.fr
}

Published by the American Physical Society under the terms of the Creative Commons Attribution 4.0 International license. Further distribution of this work must maintain attribution to the author(s) and the published article's title, journal citation, and DOI. inhomogeneous coefficients can be deduced by looking at the topological properties of the much simpler dual bulk problem. In the literature on coastal waves, a number of impressive analytical results exist in one-dimensional configuration, assuming a straight coastline and bottom topography variations perpendicular to the coast $[3,4]$. Exhibiting topological features in this context allows one to predict which features of those one-dimensional spectra are robust to coastal deformations or to fluctuations of topography along the coast direction. This method has already been proven extremely useful in condensed matter, photonics, acoustics, or mechanics [5]. Here we show how these concepts can be transferred to coastal dynamics. A major difficulty comes from the fact that fluids are continuous media, by contrast with usual condensed matter systems admitting a lattice structure. This fundamental difference prohibits a straightforward application of bulkboundary correspondence theorems that make use of this lattice structure.

Recently, the existence of two unidirectional shallow water wave modes trapped along the equator has been related to a topological invariant through a bulk-interface correspondence [6], that can now be understood as a manifestation of the Atiyah-Singer index theorem [7]. More precisely, two modes of the equatorial wave spectrum transit from one wave band to another when the zonal (eastward direction) wave number $k_{x}$ is varied. This spectral flow of two modes has been related to a (monopole) Chern number $\mathscr{C}=2$ that characterizes bulk eigenmodes twisting around a band-crossing point in parameter space $\left(k_{x}, k_{y}, f\right)$, with $k_{y}$ the meridional (northward direction) wave number, and $f$ the Coriolis parameter, that is twice the projection of the planet's angular velocity onto the local vertical axis [6]. The index $\mathscr{C}$ describes how bulk properties are changed when $f$ is varied and changes sign. It thus yields topological information about the interface wave problem defined by a change of sign of $f$. This equatorial interface Chern number cannot be used to discuss coastal problems, as the Coriolis parameter 
is held fixed in those problems: coastal Kelvin waves and equatorial Kelvin waves belong to two different classes of problems.

In condensed matter, wave problems involving the bulkboundary correspondence usually make use of another topological invariant, that we denote here as the bulk Chern number $C$. This bulk invariant is defined independently on each side of the interface between different materials that own a spectral gap. In the equatorial case, this would amount to compute a bulk Chern number for each hemisphere. In this framework, the interface Chern number $\mathscr{C}$ of the equator would just be given by the difference of bulk Chern numbers in each hemisphere, for each wave band. The existence of a bulk Chern number $C$ computed for a given value of $f$ would then be suited to address the coastal problem. It turns out that this strategy is doomed, owing to the continuous nature of fluids. Indeed, the bulk Chern number $C$ is well defined for the wave bands of a two-dimensional condensed matter system admitting an underlying lattice structure that makes compact the two-dimensional (2D) parameter space $\left(k_{x}, k_{y}\right)$ over which this topological index is computed. This structure is lost in continuous media such as fluids, so that $C$ is in general ill-defined for eigenmodes parameterized on the plane $\left(k_{x}, k_{y}\right)$. Formally, a regularization parameter can be introduced to fix this problem [8,9], but its introduction cannot be justified from first principles in the geophysical context. In addition, the bulk-boundary correspondence must be carefully stated to be valid in that case, since the value of the bulk Chern number $C$ does not necessarily correspond to the number of states that fill the gap in continuous media with a boundary $[10,11]$.

To bypass this difficulty, we follow in this paper a different strategy by showing that coastal Kelvin waves can be apprehended as an interface problem at fixed $f$ but with a varying bottom topography. We demonstrate their topological origin through the computation of an interface monopole Chern number $\mathscr{C}=1$ associated to a two-band crossing point for bulk waves in parameter space. We introduce for that purpose a new parameter: the relative local gradient of bottom topography denoted $\beta_{t}$, whose importance has recently been highlighted in other shallow water wave transport problems [12]. A change of sign of this parameter allows us to interpret the coast as an interface rather than a hard boundary. This makes possible the use of bulk-interface correspondence machinery in $\left(k_{x}, k_{y}, \beta_{t}\right)$ parameter space, at fixed $f$. The historical Kelvin wave for a hard-wall boundary condition is then recovered as a limiting case of our theory, and other classes of coastal waves can be predicted and classified using this method.

The paper is organized as follows. We recall in Sec. II a useful solvable case of rotating shallow water waves with varying bottom topography, and introduce the concept of spectral flow. We propose in Sec. II a new formulation of the model on a convenient vectorial form to study topological properties of bulk eigenmodes. We show in Sec. III that these topological properties explain a variety of spectra obtained for different problems in coastal dynamics. Details on the actual computation of the Chern number of two-band degeneracy points are recalled in the Appendix.

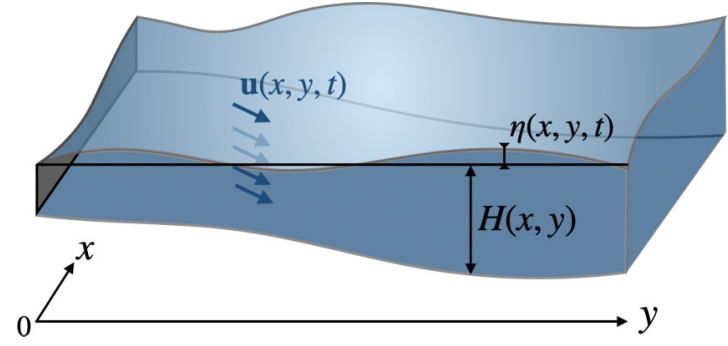

FIG. 1. Shallow fluid layer with a varying bottom topography $H(x, y)$. A perturbation around a state of rest induces a small elevation $\eta(x, y, t)$. The horizontal velocity field $\mathbf{u}(x, y, t)$ (blue arrows) is uniform in the vertical direction.

\section{SHALLOW WATER MODEL FOR COASTAL WAVES}

\section{A. Shallow water model with bottom topography}

We consider a rotating shallow water model with varying bottom topography, that describes the dynamics of a thin fluid layer with homogeneous density [4,13], as sketched in Fig. 1. In this approximation, the fluid is hydrostatic along the vertical direction, and the horizontal velocity field $\mathbf{u}(x, y, t)=$ $(u(x, y, t), v(x, y, t))$ is depth independent. The layer thickness at a given point $(x, y)$ is $h=\eta+H(x, y)$ with $\eta(x, y, t)$ the interface elevation around a state of rest. The bottom topography is thus encoded in the field $H(x, y)$. The shallow water dynamics is derived in the absence of dissipation effects using momentum conservation in the horizontal direction and mass conservation. The linearized dynamics around a state of rest is described by

$$
\partial_{t}\left(\begin{array}{l}
u \\
v \\
\eta
\end{array}\right)=\left(\begin{array}{ccc}
0 & f & -g \partial_{x} \\
-f & 0 & -g \partial_{y} \\
-\partial_{x}(H \cdot) & -\partial_{y}(H \cdot) & 0
\end{array}\right)\left(\begin{array}{l}
u \\
v \\
\eta
\end{array}\right),
$$

where $f$ is the Coriolis parameter and $g$ is the standard gravity constant at the surface of the planet. Time unit is chosen in the remainder of this paper such that $g=1$.

We review in the next section important previous results obtained in the 1960s for particular bottom topography profiles. This allows us to introduce some standard terminology used in coastal dynamics, and to set the stage for a classification of coastal waves in a much more general framework, using tools from topology.

\section{B. A solvable case for coastal waves with varying bottom topography}

Depending on the topography profile at hand, a variety of wave spectra satisfying Eq. (1) have been previously described both analytically and numerically, together with some experimental observations of the corresponding waves (see, e.g., $[3,4,14,15]$, and references therein). In particular, Ball $[16,17]$ obtained analytical results in the case of a continental shelf

$$
H(y)=H_{0}\left(1-e^{-y / a}\right)
$$

for $y>0$. The resulting spectrum is plotted in Fig. 2. The results obtained in this particular case are generic to coastal problems where half a flat-bottom $f$ plane is connected to 


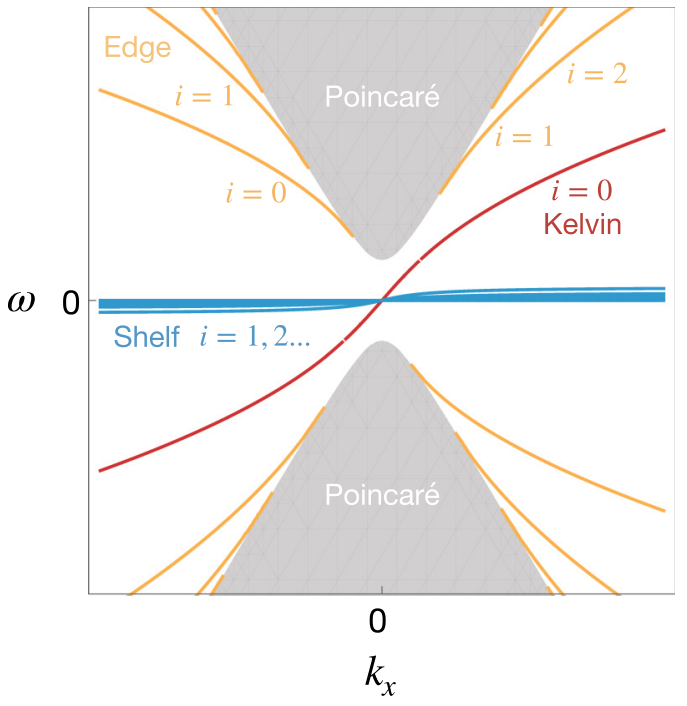

FIG. 2. Dispersion relation of coastal rotating shallow water waves with an exponential ocean depth as in Eq. (2), following [16]. This result is generic to other bottom topography profiles with a shore line $h(0)=0[3,4,14]$. "Edge" refers to inertial-gravity trapped waves at the coast. "Shelf" refers to continental shelf waves trapped at the coast. The index $i$ gives the number of nodes in the wave amplitude. The gray region corresponds to the continuous spectrum of delocalized inertia-gravity Poincaré modes. The spectral flow across the frequency gap is one, and corresponds to the presence of the Kelvin wave.

a shore line $[14,15,17]$. Those configurations exhibit both a discrete spectrum associated with trapped waves and a continuous spectrum associated with delocalized bulk waves.

The nonzero frequency bulk waves are akin to those computed on an unbounded flat-bottom $f$ plane, and are therefore referred to as the Poincaré continuum. This corresponds to two symmetric wave bands satisfying $\omega= \pm \sqrt{c^{2} k_{x}^{2}+c^{2} k_{y}^{2}+f^{2}}$, with $\left(k_{x}, k_{y}\right)$ the wave number of the bulk waves and $c=$ $\sqrt{g H_{0}}$ the phase speed of nonrotating shallow water gravity waves. Those waves are also called inertia-gravity waves, since they are surface waves influenced by rotation.

In the case of a continental shelf with the exponential profile (2), Ball found three additional classes of trapped waves. In the remainder of this paper, we shall follow the terminology used in [14] for those waves:

(1) Edge waves: Inertia-gravity waves that are trapped along the coast. Their frequency is always larger than the inertial one, $f$. Edge waves are indexed by $i \in \mathbb{N}$ for $k_{x}<0$ and by $i \in \mathbb{N}^{*}$ for $k_{x}>0$, with the index giving the number of nodes in the wave amplitude for the interface height variation.

(2) Continental shelf waves: Geostrophic modes trapped along the coast. Those shelf waves are indexed by $i \in \mathbb{N}^{*}$ (whatever the value of $k_{x}$ ), with $i$ giving the number of nodes in the wave amplitude for the interface height variation. Geostrophy means a balance between Coriolis force and pressure forces on the horizontal.

(3) Kelvin wave: Unidirectional wave whose frequency varies from $-\infty$ to $+\infty$ when increasing $k_{x}$ over the same range. Its dispersion relation thus fills the frequency gap between the negative and positive Poincaré wave bands. The amplitude of the corresponding eigenmodes does not have any node. Following the convention for other edge waves, it is indexed by $i=0$.

\section{Hard-wall spectrum as a limiting case}

Textbooks in geophysical fluid dynamics usually present coastal waves by following the original work by Kelvin where the eponymous trapped wave is computed by considering a flat-bottom $f$-plane problem with a lateral wall (see, e.g., [13]). This boundary imposes an impermeability constraint expressed as a condition of vanishing velocity across the wall. No other trapped wave than the Kelvin wave is found in this problem. In particular, there is neither inertia-gravity edge waves nor shelf waves.

Consistently, it was noticed by Ball that this original Kelvin's spectrum is recovered in the limit $a \rightarrow 0$ of an abrupt edge arbitrarily close to the vertical, for any given finite range of wave number $k_{x}$ in the direction along the wall. In this limit, the trapped shelf modes asymptote to the flat geostrophic continuum, the inertia-gravity edge modes have frequencies that tend to infinity, and the only remaining trapped mode with finite frequency is the Kelvin wave that connects the negative frequency Poincaré wave band to the positive frequency Poincaré wave band.

\section{Spectral flow}

Whatever the model for the coast, the dispersion relation of the Kelvin wave crosses the frequency gap between the geostrophic $(\omega=0)$ and the Poincaré $(|\omega|>f)$ continuum when varying $k_{x}$. This transition of a mode from one wave band to another when a parameter is varied is called a spectral flow.

In the following, we argue that this spectral flow of Kelvin mode is a manifestation of the Atiyah-Singer theorem, which, in very loose terms, relates this property to a topological index for bundles of complex eigenmodes of a dual matrix problem that is much simpler to solve than the original linear wave operator $[7,18]$. This simpler problem is referred to as the bulk problem in the following.

\section{BULK TOPOGRAPHIC SHALLOW WATER WAVES}

\section{A. The bulk problem: Symbol of the shallow water wave operator}

To define the bulk problem, we use a standard mapping between operators and their symbols provided by Weyl calculus [19]. Simply put, the symbol $H_{\text {bulk }}$ of an operator $\mathcal{H}_{\text {op }}$ for a multicomponent wave problem such as in Eq. (1) is a matrix that is obtained through a Wigner transform of the operator. Similarly, it is possible to define an operator from a symbol, using for instance Weyl calculus. (See [7,12] for details and previous use of these transformations in the context of shallow water dynamics.) In this framework, we get the following correspondence between derivatives and wave numbers:

$$
\underbrace{c(y) \partial_{y}}_{\text {Operator }} \leftrightarrow \underbrace{i k_{y} c(y)-\frac{1}{2} \partial_{y} c .}_{\text {Symbol }}
$$


The study of symbols is at the heart of microlocal analysis that relates the solutions of partial differential equations to ray tracing. Those tools were initially developed in the context of quantum physics and semiclassical analysis (see, e.g., [20]). This formalism has recently been proven fruitful in geophysical fluid dynamics; it has been used to prove the topological origin of equatorial waves [7], to explain the generic emergence of internal wave attractors in bounded stratified fluids [21], and to describe wave transport properties in geophysical flows [12]. This last application addressed specifically rotating shallow water waves with bottom topography. Our contribution is to unveil the role of topology in this problem.

Before applying Weyl calculus, it is convenient to express the shallow water dynamics (1) as

$$
\begin{gathered}
\partial_{t} \psi=-i \mathcal{H}_{\mathrm{op}} \psi, \quad \psi \equiv(\sqrt{H} u, \sqrt{H} v, \sqrt{g} \eta)^{t}, \\
\mathcal{H}_{o p} \equiv i\left(\begin{array}{ccc}
0 & f & -c \partial_{x} \\
-f & 0 & -c \partial_{y} \\
-c \partial_{x} & -c \partial_{y}-2 \beta_{t} & 0
\end{array}\right),
\end{gathered}
$$

where we have introduced the $y$-dependent wave phase speed and the relative gradient of bottom topography

$$
c \equiv \sqrt{g H}, \quad \beta_{t} \equiv \frac{1}{4} c \frac{\partial_{y} H}{H} .
$$

To simplify the presentation, we restrict ourselves to a straight coast, with topography variations in the $y$ direction only. Topologically protected features obtained in this one-dimensional case will be robust to smooth topography variations in the other direction [5].

Using Eq. (3) given by Weyl calculus, the symbol associated to the multicomponent linear wave operator (5) is the Hermitian matrix

$$
H_{\text {bulk }} \equiv\left(\begin{array}{ccc}
0 & i f & c k_{x} \\
-f & 0 & c k_{y}+i \beta_{t} \\
c k_{x} & c k_{y}-i \beta_{t} & 0
\end{array}\right) .
$$

Finding the eigenmodes $\hat{\psi}$ and eigenvalues $\omega$ of this matrix for a given set of parameters $k_{x}, k_{y}, \beta_{t}, c$ amounts to computing the (dual) bulk problem of rotating shallow water waves with varying bottom topography. This bulk problem is uniquely defined within the framework of Weyl calculus. The corresponding eigenmodes are called bulk waves, as they can be interpreted as plane wave solutions of an unbounded wave problem where $\beta_{t}$ and $c$ are held constant, with $i k_{x}, i k_{y}$ replaced by $\partial_{x}, \partial_{y}$. As explained below, global properties of the shallow water wave spectrum with varying topography is encoded in the topological properties of the symbol (7).

\section{B. Dispersion relation and discrete symmetries}

Before discussing shallow water wave spectrum associated with a profile $\beta_{t}(y)$, and its relation with a coast, let us first focus on the bulk problem, assuming $c$ constant, and $\left(k_{x}, k_{y}, \beta_{t}, f\right)$ taken as a set of free parameters.

The eigenmodes of the symbols correspond to three wave bands with frequencies satisfying

$$
\omega^{3}-\omega\left(c^{2} k_{x}^{2}+c^{2} k_{y}^{2}+f^{2}+\beta_{t}^{2}\right)+2 f \beta_{t} c k_{x}=0 .
$$

These wave bands are plotted in Fig. 3(a). In the flatbottom case $\left(\beta_{t}=0\right)$ without rotation $(f=0)$, the three wave bands consist of one zero-frequency flat band and two symmetric dispersionless mode gravity wave modes of frequency $\pm \omega$ and of phase speed $c$. Those modes touch each other at $\left(k_{x}, k_{y}\right)=(0,0)$. In the unbounded $f$-plane case $(|f|>0)$, with a flat bottom $\left(\beta_{t}=0\right)$, a spectral gap of amplitude $|f|-$ the inertial period-separates the flat band from the gravity wave modes. In that case, the flat band modes are called geostrophic modes. The gravity wave modes influenced by rotations are called Poincaré or inertia-gravity wave modes. In the $f$-plane situation $(f \neq 0)$ with a gradient of bottom topography $\left(\beta_{t} \neq 0\right)$, the flat band acquires some dispersion. Those low frequency waves bear strong similarities with planetary Rossby waves encountered on a flat-bottom ocean with a varying Coriolis parameter. We therefore identify these modes as topographic Rossby waves [3,4].

At the critical value $\left|\beta_{t}\right|=|f|$, the topographic Rossby band touches the Poincaré band, leading to a twofold degeneracy point around which the dispersion relation is linear. This is the key observation that will allow us a topological analysis of the coastal Kelvin modes in the next section. The degeneracy points are systematically obtained by vanishing the discriminant of Eq. (8), which leads to the conditions $k_{y}=$ 0 and $c^{2} k_{x}^{2}=f^{2}=\beta_{t}^{2}$. This defines the subspace of twofold degenerate eigenstates as lines in $\left(k_{x}, k_{y}=0, \beta_{t}, f\right)$ parameter space, as shown in Fig. 3(c). These lines intersect each other at the origin $\left(k_{y}, k_{x}, f, \beta_{t}\right)=(0,0,0,0)$. This intersection corresponds to the threefold waveband crossing point visible in the dispersion relations of Fig. 3(a) [6]. Here, by introducing a new bottom topography parameter, we describe the consequences of the degeneracy lines associated with twofold degeneracy points, and discuss applications to coastal waves.

Figure 3(a) reveals a striking parallel between the rotating shallow water model with a topography gradient and the celebrated Haldane model for Chern insulators [22]. This toy model, which turned out to be a building block of various topological materials, is based on a graphene lattice model for electrons. While the dispersion relation of graphene is known to show band crossings, called Dirac points, the Haldane model introduces additional terms that break either time-reversal or mirror symmetry. This symmetry breaking leads to two different gap opening mechanisms associated to two distinct topological phases. Similarly, here, the Coriolis parameter $f$ breaks time-reversal symmetry and the topography gradient parameter $\beta_{t}$ breaks mirror symmetry in the $y$ direction. An important difference with the Haldane model is that the $\left(f, \beta_{t}\right)$ diagram in Fig. 3(a) is not a topological phase diagram as one cannot assign a bulk Chern number $C$ at each point. In contrast, the topological aspect of our continuous model is expressed with a monopole Chern number $\mathscr{C}$ obtained by considering a variation of either $f$ or $\beta_{t}$ around a band-crossing point, which occurs at $f=$ $\beta_{t}$ [dashed line in Fig. 3(a)]. In particular, for the coastal Kelvin wave we focus on, one needs to consider a variation of $\beta_{t}$ at fixed $f$. Therefore, coastal Kelvin waves result from a concomitant breaking of both time-reversal and mirror symmetry. 


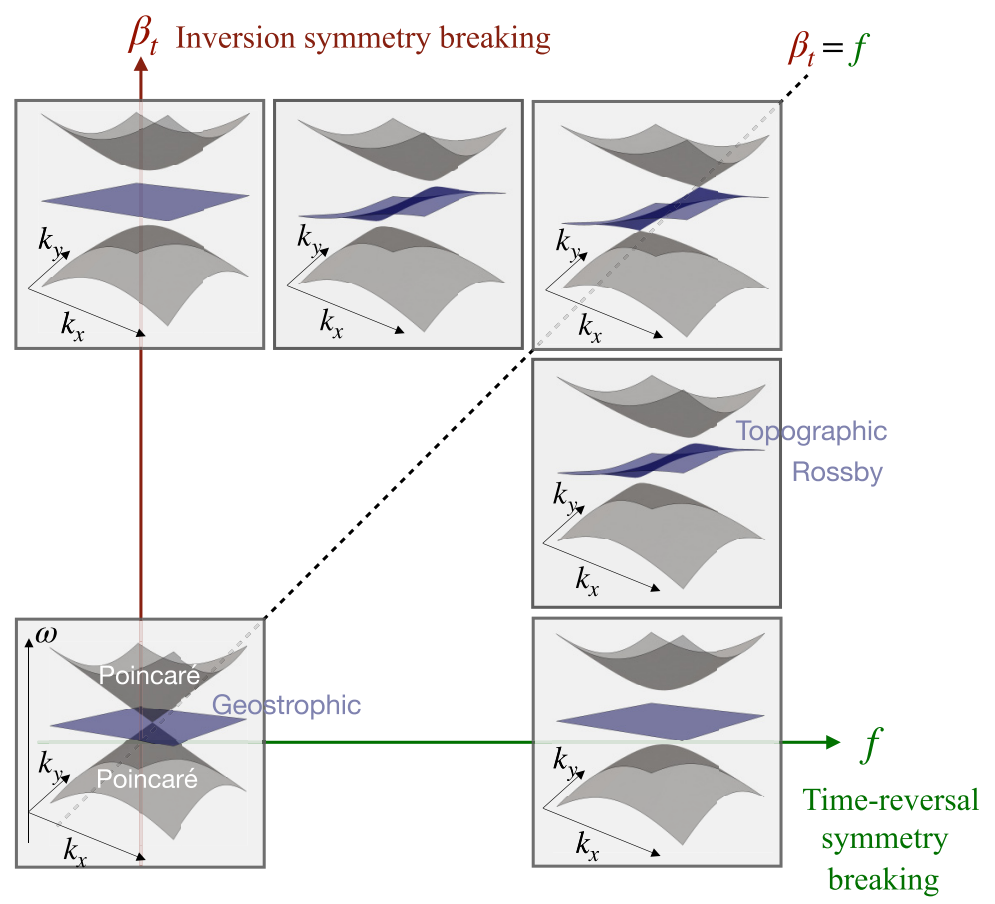

(a)

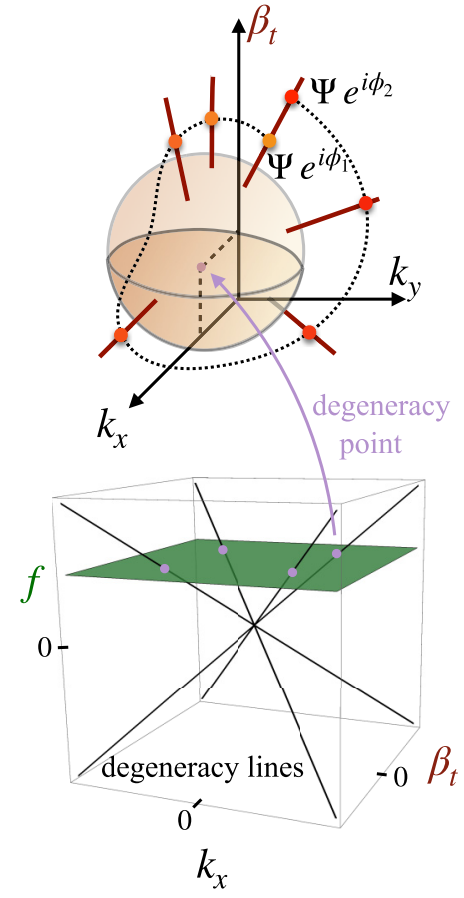

(b)

FIG. 3. (a) Dispersion relations of the bulk problem (7). The frequency gap between Poincaré wave bands and the topographic Rossby wave bands closes when $\beta_{t}= \pm f$, at $\left(k_{x}, k_{y}\right)=( \pm f / c, 0)$. (b) The upper panel sketches the vector bundle of shallow water complex eigenmodes of the matrix (7), embedded in $\left(k_{x}, k_{y}, \beta_{t}\right)$ parameter space. These eigenmodes are denoted $\Psi$. Once normalized, they are parametrized on a surface that encloses a double degeneracy point and have a phase freedom $\phi$ at each point of this surface. The impossibility to smoothly define this phase over the sphere is a topological property of the vector bundle encoded through the interface Chern number $\mathscr{C}$. Lower panel: In the coastal case, there are four such degeneracy points $\left(k_{x}, k_{y}, \beta_{t}\right)=( \pm f / c, 0, \pm f)$ to be considered, that result from the intersection of the double degeneracy lines of the bulk problem with the $f \neq 0$ constant plane.

\section{Topology of eigenmodes around degeneracy points}

The rotating shallow water model with a gradient of topography possesses topological properties associated to the aforementioned twofold degeneracy points. Each of these crossing points, when considered as isolated points in a threedimensional parameter space, are associated with a set of monopole Chern numbers $\mathscr{C}_{n} \in \mathbb{Z}$, where $n$ is the wave band index. In the coastal case we focus on, $f$ is fixed. The threedimensional parameter space to be considered is $\left(k_{x}, k_{y}, \beta_{t}\right)$, as depicted in Fig. 3(b). It will convenient to use the more abstract notation $\lambda=\left(\lambda_{1}, \lambda_{2}, \lambda_{3}\right)$ for this parameter space in the following paragraph.

The Chern numbers $\mathscr{C}_{n} \in \mathbb{Z}$ describe the obstructions to smoothly define the arbitrary global phase of the normalized eigenstates $\Psi_{n}$ of the bulk problem (7) parametrized on a closed surface $\Sigma$ that encloses the degeneracy point in parameter space $\lambda$. In Fig. 3(b), this surface is depicted as a sphere centered on one of the degeneracy points. For a given wave band $n$, the first Chern number is a global property of the eigenmode bundle defined by the closed base space $\Sigma$ and the set of eigenmodes $\Psi_{n}$ defined up to a phase on this base space [18]. It is an integer that somehow describes how twisted the eigenmodes of a given wave band are on the close surface $\Sigma$. This number can be computed through a generalization of
Gauss-Bonnet formula as

$$
\mathscr{C}_{n}=\frac{1}{2 \pi} \int_{\Sigma} \mathbf{F}^{(n)} \cdot d \boldsymbol{\Sigma},
$$

where $\mathbf{F}^{(n)}=\left(F_{\lambda_{2}, \lambda_{3}}^{(n)}, F_{\lambda_{3}, \lambda_{1}}^{(n)}, F_{\lambda_{1}, \lambda_{2}}^{(n)}\right)$ is the Berry curvature, defined as

$$
F_{\lambda_{p}, \lambda_{q}}^{(n)}=i\left(\frac{\partial \Psi_{n}^{\dagger}}{\partial \lambda_{p}} \frac{\partial \Psi_{n}}{\partial \lambda_{q}}-\frac{\partial \Psi_{n}^{\dagger}}{\partial \lambda_{q}} \frac{\partial \Psi_{n}}{\partial \lambda_{p}}\right)
$$

with the standard inner scalar product $\Psi_{n}^{\dagger} \Phi_{n}=\sum_{j=1} \Psi_{n j}^{*} \Phi_{n j}$ where $\Psi_{n j}$ is the $j$ th component of $\Psi_{n}$.

In fluid context, this formula was previously used to compute nontrivial Chern numbers of shallow water eigenmode bundles enclosing the triple degeneracy point at the origin of $\left(k_{x}, k_{y}, f\right)$ space [6]. It is tempting to interchange the roles of $f$ and $\beta_{t}$, by considering instead the monopole Chern numbers associated to the triple degeneracy point at the origin of the $\left(k_{x}, k_{y}, \beta_{t}\right)$ space. In that case, we find that the Chern numbers of the three wave bands vanish. The topography parameter alone opens a gap but does not induce nontrivial topology on eigenmode bundles. To find nontrivial topological properties, we need to consider $f \neq 0$. This is the reason why we focus here on the Chern number of twofold degeneracy points in $\left(k_{x}, k_{y}, \beta_{t}\right)$-parameter space. Their analytical computation 
through Eq. (9) requires in principle the expression of the eigenmodes of (7), which can be quite involved for a threeband problem. This difficulty is bypassed here by focusing on the absolute value of $\mathscr{C}$ only. Since we deal with two-band crossing points around which the dispersion relation is linear, we get $|\mathscr{G}|=1$ (see Appendix). We explain below how this number is related to the global shape of shallow water wave spectra with spatially varying bottom topography.

\section{SPECTRAL FLOW ALONG COASTS, ABYSS, AND ESCARPMENTS}

We now argue that the topological properties of bulk degeneracy points can be used to explain the global shape of Ball's spectrum for coastal waves shown in Fig. 2, and more generally to predict spectra for any kind of shallow water problems with varying bottom topography. We start by recalling several important mathematical results connecting the monopole Chern number $\mathscr{C}$ to spectral flow in dual interface problems.

\section{A. From degeneracy points to spectral flow}

We now consider solutions of the wave equation (1) in a case where the topography $H(y)$ varies spatially. Consequently, both the parameters $\beta_{t}(y)$ and $c(y)$ vary with $y$. When $\beta_{t}(y)$ is a monotonic function that goes through a critical [23] latitude $y_{c}$ such that a degeneracy point exists for bulk waves in $\left[k_{x}, k_{y}, \beta_{t}\left(y_{c}\right)\right]$ plane, one gets an interface problem at $y_{c}$.

The existence of degeneracy points between bands with nontrivial topological properties for the bulk eigenmodes in parameter space $\left(k_{x}, k_{y}, \beta_{t}\right)$ manifests itself in the interface wave problem as a spectral flow: some of the modes transit from one wave band to another when $k_{x}$ is varied [7]. The number of modes that transit from one band $\omega_{-}$to another one, $\omega_{+}$, close to the degeneracy point, is (algebraically) equal to the monopole Chern number $\mathscr{C}_{+}=-\mathscr{C}_{-}$associated with this degeneracy point. This method has been proven useful to interpret molecular spectra [24], to show the topological origin of equatorial [6] and Lamb-like waves in compressible stratified fluids [25], plasma [26,27], and active matter flows [28,29], and to predict new electromagnetic modes in gyrotropic media [30].

The state that transits from one band to another is trapped close to the critical value $y_{c}$ where the value of $\beta_{t}$ reaches the degeneracy point. The trapping length scale is generally given by an intrinsic length of the problem that plays the role of $\hbar$ in semiclassical analysis. In the present case, this trapping length scale is the Rossby radius of deformation $c / f$.

When the profile $\beta_{t}(y)$ is associated with several degeneracy points, one can interpret qualitatively the global shape of the spectrum by considering these degeneracy points independently from each other, provided that the trapping length scale $c / f$ is much smaller than the distance between two critical latitudes $y_{c, i}$ and $y_{c, i+1}$ associated with different degeneracy points between the two bands.

For a given degeneracy point, the sign of the spectral flow changes with the sign of $d \beta_{t} / d y$. In other words, a state that transits from the lower band to the upper band when $\beta_{t}$ is an increasing function of $y$ would transit from the upper band to the lower band if $\beta_{t}(y)$ was a decreasing function of $y$ that crosses the same degeneracy point. When a given degeneracy point is crossed several times by the profile $\beta_{t}(y)$ over a distance smaller than the trapping length scale, two cases need to be considered, depending on the number of crossing points. If this number is even, then there is no spectral flow. If this number is odd, then the direction of the spectral flow is given by the one deduced from the first crossing point.

Thus, topology provides a toolbox that makes possible a classification of coastal waves, when properly recast as an interface problem. In the following, we use this toolbox to interpret three classes of coastal spectra solved numerically using Dedalus software [31]. The results are summarized in Fig. 4.

\section{B. Topological origin of the coastal Kelvin wave}

The profile $\beta_{t}(y)$ of the relative topography gradient parameter associated with the exponential Ball's bottom topography profile $H(y)$ of Eq. (2) is shown in Fig. 4(a). The key point is that $\beta_{t}(y)$ is decreasing from $y=0$ to $y \rightarrow+\infty$, and goes through the degeneracy point $\beta_{t}=f$ with $\beta_{t}^{\prime}<0$. We have thus recast the coastal problem into an interface problem.

As expected from our analysis of the bulk problem, and from general results on the correspondence between such bulk indices and spectral flow for the dual interface problems, we observe that the spectrum of Fig. 4(a) exhibits one state that transits from the lower frequency Poincaré wave band to the upper one. This shows the topological origin of the coastal Kelvin wave. This topological property distinguishes the coastal Kelvin wave from the other trapped boundary waves, namely, edge and shelf waves, as it guarantees its existence against continuous deformations of the topography profile and constrains its dispersion relation to fill the frequency gap.

We stress that the interface is not the coast itself (at $y=0$ ); it is rather the critical point $y_{c}$ where $\beta_{t}\left(y_{c}\right)=f$. In the limit $a \rightarrow 0$, this critical point becomes closer to the coast: $y_{c} \rightarrow 0$.

Depending on the detailed shape of $H(y)$, they could actually be an odd number of degeneracy points, but the difference between degeneracy points associated with $\beta_{t}^{\prime}<0$ and degeneracy points associated with $\beta_{t}^{\prime}>0$ will always be one. We also notice that the intrinsic length scale vanishes close to the coast as $c(y) / f \sim y^{1 / 2}$. Qualitatively, this guarantees that the actual shore line is screened from the interior dynamics.

\section{Topographic Yanai waves at an interface with deep waters}

In the previous case, we found that one mode is gained by the upper band when $k_{x}$ is increased, and related this spectral flow to a profile $\beta_{t}(y)$ crossing the degeneracy point from above. We expect an opposite spectral flow when the profile $\beta_{t}(y)$ crossed the same degeneracy point from below. That is to say, we expect a net loss of one mode in the upper band when $k_{x}$ is increased. This is the situation depicted in Fig. 4(b), with the topography profile $H(y)=H_{0}\left(1+3 e^{-\left[\left(y_{a}-y\right) / 0.1\right]}\right)$. This profile is representative of cases where half a flat-bottom $f$ plane opens to infinitely deep water (the abyss).

As shown in the spectrum displayed in Fig. 4(b), there is indeed a mode that transits from the upper Poincaré band 
(a) Coast to flat bottom
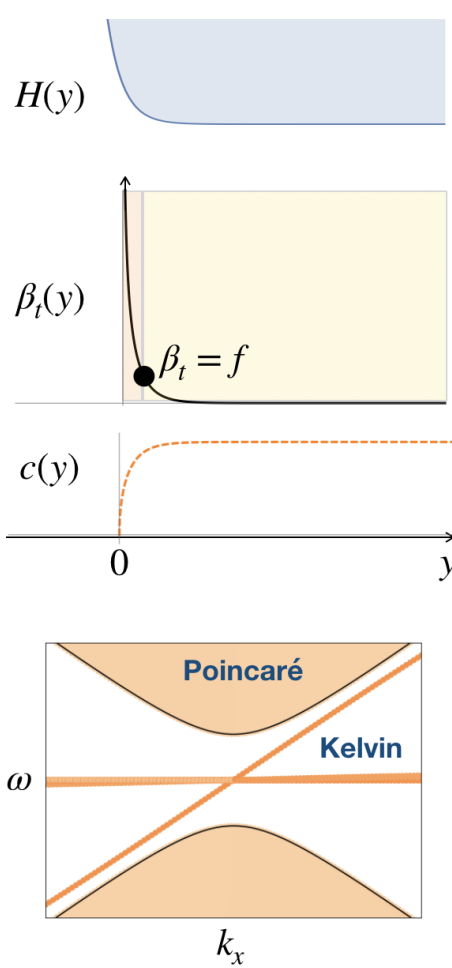

(b) Flat bottom to abyss
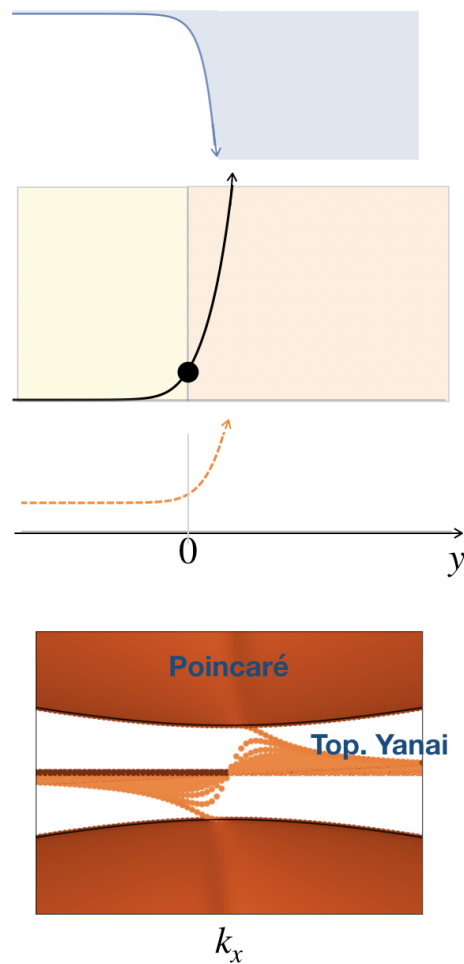

(c) Escarpment
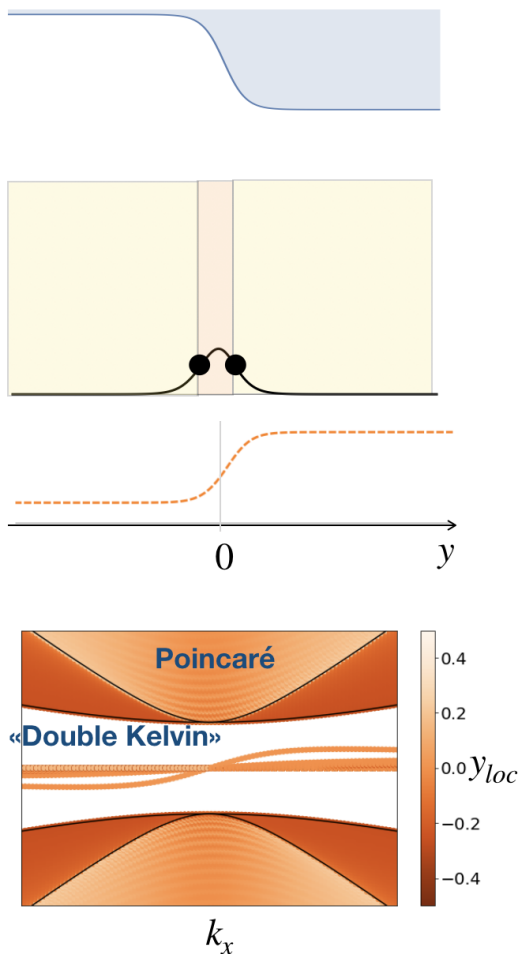

FIG. 4. Three topologically distinct interface problems. (a) The case of a coast, where $\beta_{t}(y)$ crosses a degeneracy point $y_{c}>0$ from above, and $c$ vanishes at $y=0$. This is related to spectral flow +1 due to the presence of the Kelvin wave in the spectrum below. (b) The case of a flat-bottom $f$ plane opened to an "abyss" region where bottom topography diverges with $y \rightarrow+\infty$. The same degeneracy point as in case (a) is crossed by the profile $\beta_{t}(y)$, but from below. This is related to spectral flow -1 due to the presence of a topographic Yanai wave. (c) The case of an escarpment separating two flat-bottom half $f$ planes with different depth. This is a topologically trivial case that can be interpreted as the concatenation of cases (b) and (a). There is a so-called double Kelvin mode in the frequency gap; this mode is not associated with a spectral flow.

to the geostrophic wave band when $k_{x}$ is increased. This topological mode is an inertial mode at $k_{x}=0$, where $\omega=f$. Its branch bears strong similarities with the equatorial Yanai mode, as it connects the topographic Rossby wave band to the inertia-gravity wave band. We therefore propose to call it a topographic Yanai mode; the existence of such modes was actually described in previous work by Iga in a classification of shallow water spectra depending on the boundary conditions in channel geometries [32]. Our study now shows that such a mode has a topological origin, just as the coastal Kelvin wave. As far as topology is concerned, the only difference between those two modes is their opposite group velocity, which can be related to the different sign of $\beta_{t}^{\prime}$ at the critical point where $\beta_{t}\left(y_{c}\right)=f$.

The topography profile $H(y)$ related to the topographic Yanai case is peculiar, as it diverges with $y$. While a number of interesting and useful results are often derived in a shallow water context with diverging bottom topography profiles, one should keep in mind that such profiles are not consistent with the hypothesis underlying the derivation of shallow water equations from more comprehensive 3D Euler dynamics. It will be interesting to ask whether topographic Yanai surface waves exist in the context of incompressible 3D Euler flows with gravity. Within the shallow water framework, the connection from a shallow coastal area to a deeper abysslike area can only be consistent with the hypothesis required for the model derivation if the abyss depth $H(y)$ tends to a constant value at large $y$. In other words, the profile $\beta_{t}(y)$ must be decreasing towards the origin at large $y$. This leads to the escarpment case discussed below.

\section{Double Kelvin waves over escarpment are topologically trivial}

An escarpment is a variation of topography separating two oceanic basins with different depths, as illustrated in Fig. 4(c). We argue below that escarpments result from the concatenation of the two topological cases described above. Since the singularities cancel each other, this leads to a configuration that is topologically trivial. This leads, however, to a nontrivial reinterpretation of the spectra associated with escarpments.

In the case of an escarpment $H(y)$ separating a shallow oceanic basin with depth $h_{1}$ to a deep oceanic basin with depth $h_{2}$, a peculiar trapped mode with frequency lower than the inertial frequency $f$, and without node in their amplitude in the $y$ direction, was found in the $1960 \mathrm{~s}$ by Longuet-Higgins [33,34]. This mode was dubbed double Kelvin wave, because its amplitude is decaying on both sides of the escarpment. Those waves do not fill the gap between geostrophic mode and Poincaré modes, as reported in Fig. 4(c). Instead, their dispersion relation is similar to Rossby waves, with a group velocity changing sign at high 
wave numbers. In particular, these trapped modes are not unidirectional.

From a topology and spectral flow perspective, the absence of modes transiting from one band to another is consistent with the profile $\beta_{t}(y)$ that either crosses twice the same degeneracy point or does not cross any degeneracy point at all when the escarpment is not sufficiently steep. The case of a double crossing of the same degeneracy point with opposite $\operatorname{sgn}\left(\beta_{t}^{\prime}\right)$ is topologically equivalent to the one without degeneracy points. The double Kelvin wave is topologically trivial. Our analysis shows that this peculiar mode can now be understood as a hybrid mode between a coastal Kelvin wave related to the first degeneracy point and a coastal topographic Yanai wave related to the second degeneracy point. The singularities associated with those two degeneracy points cancel each other. In other words, the double Kelvin mode of case (c) in Fig. 4 is a footprint of the concatenation of $\beta_{t}$ profiles described in cases (a) and (b). As such, those hybrid modes could rather be dubbed mixed topographic Yanai-Kelvin waves. By this way, topology gives a complementary point of view to [32] on this problem.

\section{CONCLUSION}

Topology makes possible a new classification of shallow water waves with varying topography. This unifies several results on coastal waves reported in previous works, e.g., $[3,4,32]$. Topology guarantees that the spectral flow reported in the case of a straight coastline and one-dimensional topography variations in the direction perpendicular to the coast are also robust to changes in coastline shape or to perturbation of the topography profile in the direction along the coastline, as observed in experiments [35].

It is worth stressing similarities and differences between the use of topology in this study and in condensed matter physics. Topology is routinely used to characterize trapped boundary modes in condensed matter or related systems admitting an underlying lattice structure, via the celebrated bulk-boundary correspondence. Strictly speaking, this correspondence cannot be applied in continuous media such as fluid systems in general, unless some regularization terms are present in the physical model, as it might be the case, e.g., in certain active fluids [8-10]. This procedure allows a rigorous investigation of the bulk-boundary correspondence, accounting for various boundary conditions beyond the impermeability one, when the dispersion relation is gapped due to the introduction of a fixed parameter that breaks a symmetry, such as $f[10,11]$.

Although the introduction of the regularization term could be meaningful in active matter systems, it is rather artificial in the geophysical realm. To avoid the use of such a mathematical trick, we found a quite natural geophysical way, in this study, to define a Chern number in the $f$ plane, by considering a parameter $\beta_{t}$ in the equation that introduces new degeneracy points in the dispersion relation in a $3 \mathrm{D}$ parameter space. The introduction of this parameter does not regularize the eigenmode vector field at infinity. Instead, it can be seen as a regularization parameter of the abrupt wall considered in $[8,10]$. Concretely, this procedure turns the coastal (boundary) problem into an interface problem, where monopole Chern numbers appear. The key difference with the usual bulkboundary correspondence being that $\beta_{t}$ is a varying parameter that changes sign, and not simply a fixed term that opens a gap. Those Chern monopoles can naturally be used in fluids problems or in other continuous media to make predictions about interface geometries (i.e., partial differential equations with spatially varying parameter, without a boundary), by means of the Atiyah-Singer index theorem. This contrasts with other demonstrations of the bulk-boundary correspondence in the condensed matter context that involve an actual (sharp) boundary, and that do not refer to the Atiyah-Singer index theorem [2,36,37].

Using this classification, it is now possible to discover new classes of "topographic-equatorial" waves, when both parameters $f$ and $\beta_{t}$ vary in the meridional direction. In particular, since the critical latitudes associated with degeneracy points are shifted by the presence of a relative topographic gradient $\beta_{t} \neq 0$, we predict that the location of trapped modes will also be shifted. This is a new example of a dynamical equator that differs from the usual equator [38]. It will be also interesting to investigate in future works the dynamics of relatively deep planetary waves close to the tangent equatorial cylinder in giant planets such as Jupiter [39]. In fact, this case bears strong similarities with the topography profile " $f$-plane to abyss" in Fig. 4(b), with possible experimental realization where the parameter $\beta_{t} / f$ has already been shown to play a central role [40].

The present work dealt with linear waves around a state of rest; this set the stage for more comprehensive studies addressing the role of topology in the presence of nonlinearities, with a possible two-way coupling between coastal boundary waves and interior dynamics [41,42].

From a fundamental perspective, the bottom line of this study is an interpretation of Kelvin's original boundary problem with an impermeable wall as a limiting case of an interface problem with a varying bottom topography. This unveils the topological origin of the unidirectional trapped mode computed by Kelvin in his seminal 1880 paper on tides, as resulting from an interplay between time-reversal and mirror symmetry breaking.

\section{ACKNOWLEDGMENTS}

We warmly thank Frederic Faure, Yohei Onuki, Nicolas Perez, and Clement Tauber for their useful insights on this problem. This project was supported by the national grant WTF ANR-18-CE30-0002-01 and Idex de Lyon Breakthrough project TORE.

\section{APPENDIX: TOPOLOGY OF EIGENMODE BUNDLES ENCLOSING TWO-BAND DEGENERACY POINTS}

We derive here a classical result on the topology of eigenmode bundles enclosing two-band degeneracy points. More details are presented in standard reviews and textbooks on the physics of topological waves. We consider here two-band crossing of an Hermitian problem parametrized by a vector $\lambda=\left(\lambda_{1}, \lambda_{2}, \lambda_{3}\right)$ that vanishes at the band-crossing point. In our case $\lambda=\left(k_{x}, k_{y}, \beta_{t, y}\right)-( \pm f, 0, \pm f)$. After a projection onto the two bands crossing each other, the two-band crossing 
problem is described by a reduced $2 \times 2$ matrix, which can always be written as

$$
H_{r}(\boldsymbol{\lambda})=\left(\begin{array}{cc}
h_{3}(\boldsymbol{\lambda})+\omega_{0} & h_{1}(\boldsymbol{\lambda})-i h_{2}(\boldsymbol{\lambda}) \\
h_{1}(\boldsymbol{\lambda})+i h_{2}(\boldsymbol{\lambda}) & -h_{3}(\boldsymbol{\lambda})+\omega_{0}
\end{array}\right),
$$

where $\boldsymbol{h}(\boldsymbol{\lambda})=\left(h_{1}(\boldsymbol{\lambda}), h_{2}(\boldsymbol{\lambda}), h_{3}(\boldsymbol{\lambda})\right) \in \mathbb{R}^{3}$. The eigenvalues of the matrix $H_{r}$ are given by $\omega_{ \pm}=\omega_{0} \pm$ $\sqrt{h_{1}^{2}(\boldsymbol{\lambda})+h_{2}^{2}(\boldsymbol{\lambda})+h_{3}^{2}(\boldsymbol{\lambda})}$ and cross at $\omega_{ \pm}=\omega_{0}$ when $\mathbf{h}=0$.

Let us for a moment forget the dependence on $\lambda$, and consider $\mathbf{h}=\left(h_{1}, h_{2}, h_{3}\right)$ as a parameter. Using Eqs. (9) and (10), it is a classical exercise to show that the Chern number of the two eigenmode bundles parametrized over a closed surface $\Sigma_{\mathbf{h}}$ enclosing the degeneracy point $\mathbf{h}=0$ are

$$
\mathcal{C}_{ \pm}^{h}=\frac{1}{2 \pi} \int_{\Sigma_{\mathbf{h}}} \mathbf{F}^{( \pm)}(\mathbf{h}) \cdot d \boldsymbol{\Sigma}_{\mathbf{h}}=\mp 1,
$$

where $\mathbf{F}^{( \pm)}$is the Berry curvature associated with eigenmodes of Eq. (A1) denoted $\Psi_{ \pm}(\mathbf{h})$. The computation is explained in detail in $[7,43,44]$. One can actually visualize the singularity associated with this nonzero Chern number by a direct inspection of the eigenmode expression, using polar coordinates $(h, \theta, \phi)$ in parameter space with $h_{1}=h \sin \theta \cos \phi$, $h_{2}=h \sin \theta \sin \phi$, and $h_{3}=h \cos \theta$ :

$$
\Psi_{+}=\left(\begin{array}{c}
e^{-i \phi} \cos \frac{\theta}{2} \\
\sin \frac{\theta}{2}
\end{array}\right), \quad \Psi_{-}=\left(\begin{array}{c}
-\sin \frac{\theta}{2} \\
e^{i \phi} \cos \frac{\theta}{2}
\end{array}\right) .
$$

Those normalized eigenmodes are defined up to an arbitrary phase factor $e^{i \alpha_{ \pm}}$. With our phase choices, the singularity in $\Psi_{ \pm}(\mathbf{h})$ occurs at $\theta=\pi$, as $\mathbf{h}$ is left invariant by changes in $\phi$, while $\Psi_{ \pm}$varies with $\phi$. One can use the phase freedom to remove the singularity from the location where $\theta=\pi$. However, this phase change would only shift the singularity elsewhere on the eigenmode bundle defined on any surface enclosing the origin $h=0$. We also see that at the singular point $\theta=\pi$, the phase factor varying with $\phi$ is opposite for $\Psi_{-}$and for $\Psi_{+}$. The Chern number $\mathcal{C}_{ \pm}^{h}$ quantifies these singularities.

Now, we want to compute the Chern number for the eigenmode bundles defined on a surface denoted $\Sigma_{\lambda}$ in $\lambda$-parameter space rather than in $\mathbf{h}$ space. We assume that the surface $\Sigma_{\lambda}$ encloses the band-crossing point $\lambda=0$. We introduce the degree $\operatorname{deg} h$ that counts how many times the application $h$ : $\lambda \in \Sigma_{\lambda} \rightarrow \boldsymbol{h} /|\boldsymbol{h}| \in \Sigma_{\boldsymbol{h}}$ wraps the unit sphere in $\mathbf{h}$-parameter space when $\lambda$ is varied over $\Sigma_{\lambda}$. A direct computation of the Chern number through the integral of Berry curvature expressed either in $\boldsymbol{\lambda}$-parameter space or $\mathbf{h}$-parameter space yields to

$$
\begin{aligned}
\mathscr{C}_{ \pm} & =\frac{1}{2 \pi} \int_{\Sigma_{\lambda}} \mathbf{F}^{( \pm)}(\lambda) d \boldsymbol{\Sigma}_{\lambda} \\
& =\frac{1}{2 \pi} \int_{h\left(\Sigma_{\lambda}\right)} \mathbf{F}^{( \pm)}(\boldsymbol{h}) d \boldsymbol{\Sigma}_{\boldsymbol{h}}=(\operatorname{deg} h) \mathcal{C}_{ \pm}^{h} .
\end{aligned}
$$

The last equality is obtained by noting that integrating the Berry curvature over a closed surface $\Sigma_{\mathbf{h}}$ enclosing $\mathbf{h}=0$ yields the same results as integration over the unit sphere in h space.

A method to find $\operatorname{deg} h$ is to consider an arbitrary vector $\mathbf{h}_{0}$, to find all the vectors $\lambda_{0}$ such that $\lambda_{0}=\mathbf{h}^{-1}\left(\mathbf{h}_{\mathbf{0}}\right)$, and to compute

$$
\operatorname{deg} h=\sum_{\lambda_{0} \in \boldsymbol{h}^{-1}\left(\mathbf{h}_{0}\right)} \operatorname{sgn}\left[\left.\operatorname{det}\left(\frac{\partial h_{j}}{\partial \lambda_{i}}\right)\right|_{\lambda_{0}}\right]
$$

(see, e.g., [45] for more details on the degree of an application). Generically, the function $\left\{h_{j}\right\}$ depends linearly on the parameters $\left\{\lambda_{i}\right\}$ when $\lambda \rightarrow 0$, i.e., when $\Sigma_{\lambda}$ is chosen sufficiently close to the degeneracy point. In that case, there is a unique vector $\lambda_{0}=h^{-1}$ to be taken into account in the sum, so that the possible values of the degree are restricted to \pm 1 . In other words, close to the degeneracy point there is a nonsingular linear transformation from $\lambda$ to $\mathbf{h}$, so that $\mathbf{h} /|\mathbf{h}|$ wraps one time the sphere $S^{2}$ when $\lambda$ wraps the surface $\Sigma_{\lambda}$, and the degree accounts for a possible change orientation induced by the linear transformation. Consequently, the Chern numbers of the eigenmode bundles enclosing the degeneracy point are the same up to a sign in $\lambda$-parameter space and h-parameter space.
[1] W. Thomson, On gravitational oscillations of rotating water, Proc. R. Soc. Edinburgh 10, 92 (1880).

[2] Y. Hatsugai, Chern Number and Edge States in the Integer Quantum Hall Effect, Phys. Rev. Lett. 71, 3697 (1993).

[3] P. H. LeBlond and L. A. Mysak, Waves in the Ocean (Elsevier, New York, 1981).

[4] V. Zeitlin, Geophysical Fluid Dynamics: Understanding (Almost) Everything with Rotating Shallow Water Models (Oxford University Press, New York, 2018).

[5] M. Z. Hasan and C. L. Kane, Colloquium: Topological insulators, Rev. Mod. Phys. 82, 3045 (2010).

[6] P. Delplace, J. Marston, and A. Venaille, Topological origin of equatorial waves, Science 358, 1075 (2017).

[7] F. Faure, Manifestation of the topological index formula in quantum waves and geophysical waves, arXiv:1901.10592.
[8] C. Tauber, P. Delplace, and A. Venaille, A bulk-interface correspondence for equatorial waves, J. Fluid Mech. 868, R2 (2019).

[9] A. Souslov, K. Dasbiswas, M. Fruchart, S. Vaikuntanathan, and V. Vitelli, Topological Waves in Fluids with Odd Viscosity, Phys. Rev. Lett. 122, 128001 (2019).

[10] C. Tauber, P. Delplace, and A. Venaille, Anomalous bulk-edge correspondence in continuous media, Phys. Rev. Res. 2, 013147 (2020).

[11] G. M. Graf, H. Jud, and C. Tauber, Topology in shallow-water waves: A violation of bulk-edge correspondence, Commun. Math. Phys. 383, 731 (2021).

[12] Y. Onuki, Quasi-local method of wave decomposition in a slowly varying medium, J. Fluid Mech. 883, A56 (2020).

[13] G. K. Vallis, Atmospheric and Oceanic Fluid Dynamics: Fundamentals and Large-Scale Circulation, 2nd ed. (Cambridge University Press, Cambridge, UK, 2017). 
[14] J. Huthnance, On trapped waves over a continental shelf, J. Fluid Mech. 69, 689 (1975).

[15] M. C. Hendershott, Ocean tides, Eos, Trans. Am. Geophys. Union 54, 76 (1973).

[16] F. Ball, Edge waves in an ocean of finite depth, in Deep Sea Research and Oceanographic Abstracts (Elsevier, New York, 1967), Vol. 14, pp. 79-88.

[17] G. Reznik and V. Zeitlin, Resonant excitation of trapped waves by Poincaré waves in the coastal waveguides, J. Fluid Mech. 673, 349 (2011).

[18] M. Nakahara, Geometry, Topology and Physics (CRC, Boca Raton, FL, 2003).

[19] M. Zworski, Semiclassical Analysis, Graduate Studies in Mathematics Vol. 138 (American Mathematical Society, Providence, RI, 2012).

[20] R. G. Littlejohn and W. G. Flynn, Geometric phases in the asymptotic theory of coupled wave equations, Phys. Rev. A 44, 5239 (1991).

[21] Y. Colin de Verdière and L. Saint-Raymond, Attractors for twodimensional waves with homogeneous Hamiltonians of degree 0, Commun. Pure Appl. Math. 73, 421 (2020).

[22] F. D. M. Haldane, Model for a Quantum Hall Effect Without Landau Levels: Condensed-Matter Realization of the "Parity Anomaly," Phys. Rev. Lett. 61, 2015 (1988).

[23] Note that this critical latitude should be confused with critical layers that occur when waves propagate in the presence of a mean flow.

[24] F. Faure and B. Zhilinskii, Topological Chern Indices in Molecular Spectra, Phys. Rev. Lett. 85, 960 (2000).

[25] M. Perrot, P. Delplace, and A. Venaille, Topological transition in stratified fluids, Nat. Phys. 15, 781 (2019).

[26] J. B. Parker, J. Marston, S. Tobias, and Z. Zhu, Topological Gaseous Plasmon Polariton in Realistic Plasma, Phys. Rev. Lett. 124, 195001 (2020).

[27] J. B. Parker, J. W. Burby, J. B. Marston, and S. M. Tobias, Nontrivial topology in the continuous spectrum of a magnetized plasma, Phys. Rev. Research 2, 033425 (2020).

[28] S. Shankar, M. J. Bowick, and M. C. Marchetti, Topological Sound and Flocking on Curved Surfaces, Phys. Rev. X 7, 031039 (2017).

[29] R. Green, J. Armas, J. de Boer, and L. Giomi, Topological waves in passive and active fluids on curved surfaces: A unified picture, arXiv:2011.12271.
[30] M. Marciani and P. Delplace, Chiral Maxwell waves in continuous media from Berry monopoles, Phys. Rev. A 101, 023827 (2020).

[31] K. J. Burns, G. M. Vasil, J. S. Oishi, D. Lecoanet, and B. P. Brown, Dedalus: A flexible framework for numerical simulations with spectral methods, Phys. Rev. Res. 2, 023068 (2020).

[32] K. Iga, Transition modes of rotating shallow water waves in a channel, J. Fluid Mech. 294, 367 (1995).

[33] M. Longuet-Higgins, Double Kelvin waves with continuous depth profiles, J. Fluid Mech. 34, 49 (1968).

[34] M. Longuet-Higgins, On the trapping of waves along a discontinuity of depth in a rotating ocean, J. Fluid Mech. 31, 417 (1968).

[35] C. Ren, X. Fan, Y. Xia, T. Chen, L. Yang, J.-Q. Zhong, and H. Zhang, Robust propagation of internal coastal Kelvin waves in complex domains, Phys. Rev. Fluids 6, L022801 (2021).

[36] G. M. Graf and J. Shapiro, The bulk-edge correspondence for disordered chiral chains, Commun. Math. Phys. 363, 829 (2018).

[37] E. Prodan and H. Schulz-Baldes, Bulk and Boundary Invariants for Complex Topological Insulators: From K-Theory to Physics, Mathematical Physics Studies (Springer, New York, 2016).

[38] J. P. Boyd, Dynamics of the Equatorial Ocean (Springer, New York, 2018).

[39] M. Heimpel, J. Aurnou, and J. Wicht, Simulation of equatorial and high-latitude jets on Jupiter in a deep convection model, Nature (London) 438, 193 (2005).

[40] S. Cabanes, J. Aurnou, B. Favier, and M. Le Bars, A laboratory model for deep-seated jets on the gas giants, Nat. Phys. 13, 387 (2017).

[41] B. Deremble, E. Johnson, and W. Dewar, A coupled model of interior balanced and boundary flow, Ocean Modell. 119, 1 (2017).

[42] A. Venaille, Quasi-geostrophy against the wall, J. Fluid Mech. 894, R1 (2020).

[43] M. Fruchart and D. Carpentier, An introduction to topological insulators, C. R. Phys. 14, 779 (2013).

[44] B. A. Bernevig and T. L. Hughes, Topological Insulators and Topological Superconductors (Princeton University Press, Princeton, NJ, 2013).

[45] B. Dubrovin, A. Fomenko, and S. Novikov, Modern Geometry-Methods and Applications: Part II: The Geometry and Topology of Manifolds (Springer Science and Business Media, New York, 1985). 